\title{
LES INCIDENCES DE L'INTÉGRATION EUROPÉENNE DE LA SLOVAQUIE SUR LES PROGRAMMES SCOLAIRES SLOVAQUES D'ENSEIGNEMENT DES LANGUES ÉTRANGÈRES
}

\author{
François SCHMITT \\ Université Matej Bel de Banská Bystrica, Slovaquie \\ francois.schmitt@umb.sk \\ Article reçu le 5 novembre 2016 | révisé depuis le 10 novembre 2016 | accepté le 23 décembre 2016
}

\begin{abstract}
RÉSUMÉ. L'influence du contexte national et de la politique linguistique éducative de l'État sur l'enseignement des langues étrangères est particulièrement marquée. En Europe, les politiques linguistiques éducatives ont aujourd'hui tendance à se conformer aux orientations du Conseil de l'Europe et de l'Union européenne qui ont mis en place des politiques linguistiques éducatives en phase avec les évolutions récentes de la mondialisation. Cette incidence des politiques linguistiques éducatives européennes est particulièrement visible dans les modifications apportées au programme d'enseignement public des langues étrangères slovaque de 2007 par rapport à celui de 1997.
\end{abstract}

Mots-clés : conseil de l'Europe, enseignement des langues étrangères, politique linguistique éducative, Union européenne.

\begin{abstract}
Domestic political situation and domestic linguistic educational policy widely influence foreign languages teaching. Nowadays linguistic educational policies in Europe tend to comply with the Council of Europe and the European Union political guidelines. These institutions have set up linguistic educational policies in line with recent evolutions of globalisation. The modifications made to the Slovak curriculum of foreign languages between 1997 and 2007 clearly show how deep can be the influence of the European linguistic educational policy on domestic linguistic educational policies
\end{abstract}

Keywords: council of Europe, European Union, foreign languages teaching, linguistic educational policy.

\section{INTRODUCTION}

Comme tout enseignement dispensé dans un cadre institutionnel, l'enseignement scolaire des langues étrangères est astreint aux prescriptions émanant $\mathrm{du}$ cadre programmatique et législatif dont il relève. Cet ensemble réglementaire est défini par un programme qui précise les objectifs de l'enseignement, les orientations didactiques et les contenus à transmettre. Ce programme est renouvelé périodiquement, non seulement pour mettre à jour les avancées didactiques et les évolutions linguistiques et culturelles des langues étrangères enseignées, mais aussi pour mettre en application les choix de l'État en matière de politique linguistique éducative. Or, dans le contexte d'une intégration européenne de 
plus en plus poussée depuis la fin du $X^{\mathrm{e}}$ siècle, les États membres de l'Union européenne sont amenés à adapter leurs politiques linguistiques éducatives aux orientations européennes dans ce domaine. Cette adaptation est particulièrement visible dans les nouveaux États membres où ces transformations ont été plus rapides qu'ailleurs. Pour rendre compte de ce phénomène, nous étudierons le cas de la Slovaquie après avoir auparavant défini la notion de politique linguistique éducative et rendu compte des principales orientations des institutions européennes en la matière.

\section{LA NOTION DE POLITIQUE LINGUISTIQUE ÉDUCATIVE DANS L'ENSEIGNEMENT DES LANGUES ÉTRANGÈRES}

Parmi les nombreux facteurs influant sur l'enseignement scolaire, le contexte national joue un rôle particulièrement important. Son incidence apparaît de manière plus ou moins explicite dans les contenus et les supports pédagogiques, mais aussi de manière plus globale dans les choix opérés en matière de politique linguistique éducative.

\section{Enseignement des langues étrangères et contexte national}

Deux types de facteurs influent sur l'enseignement des langues étrangères: d'une part, la situation politique, économique et sociale du pays ; d'autre part, l'époque.

\section{L'incidence du contexte national}

Partant de l'hypothèse selon laquelle « la relation entre langue et culture étrangères dépend du contexte national où la langue est enseignée", Zarate (2010, p.11) met en évidence l'influence du contexte national sur la conception des méthodes locales de français langue étrangère. Elle souligne en particulier le poids «idéologique» du pays d'enseignement dans l'enseignement des langues étrangères en milieu scolaire dans la mersure où l'école se donne aussi bien pour tâche d'ouvrir le jeune apprenant à la langue-culture enseignée que de veiller à sa construction identitaire impliquant la mise en valeur de son pays à travers l'enseignement des langues étrangères. C'est particulièrement le cas lorsque la langue-culture étrangère enseignée appartient «à la même aire idéologique » (Zarate, 2010, p.20) que celle de l'apprenant. Dans ce cas, l'image positive de la langue-culture enseignée contribue à donner une image positive à l'apprenant de son propre pays. L'enseignement des langues étrangères répond donc aux orientations politiques et sociales $\mathrm{du}$ pays d'enseignement. Pour cette raison, cet enseignement correspond aussi aux enjeux de son temps.

\section{L'incidence de l'époque}

Puren (1989, p. 9-12) a montré que les choix pédagogiques en matière $\mathrm{d}$ 'enseignement des langues étrangères étaient liés aux enjeux politiques de leur époque. Il explique ainsi l'introduction de la méthode directe en France au tournant des XIXe et XXe siècles par la volonté des républicains au pouvoir de promouvoir une méthode adaptée à la formation de citoyens autonomes et libres de penser par euxmêmes, contre la méthode classique fondée sur l'enseignement du latin défendue par les royalistes et les conservateurs catholiques.

En Slovaquie, l'évolution politique a encore davantage pesé sur l'enseignement des langues étrangères qu'en France, en particulier en ce qui concerne le choix des langues enseignées. Ainsi, le russe, première langue étrangère obligatoire sous le régime communiste, a été largement marginalisé dans les années 1990 au profit des langues de l'Ouest avant de retrouver un certain regain depuis quelques années. Les choix politiques continuent cependant aujourd'hui encore de peser de tout leur poids sur l'enseignement des langues étrangères en Slovaquie comme le montre, par exemple, la décision depuis 2011 de rendre l'enseignement de l'anglais obligatoire comme première langue étrangère (Baranová, sous presse). L'enseignement des langues étrangères représente donc un enjeu de pouvoir important et, pour cette raison, occupe une 
place de choix dans les politiques linguistiques.

\section{Politique linguistique et politique linguistique éducative}

On distingue, d'une part, la politique linguistique comme action du pouvoir sur la langue et son usage et, d'autre part, la politique linguistique éducative comme action du pouvoir sur l'enseignement des langues.

\section{La politique linguistique}

Lorsque le pouvoir politique agit directement sur la langue, en changeant, par exemple, l'écriture comme ce fut le cas de la réforme de la langue turque en 1928 ou en réformant l'orthographe, on parle de «planification de corpus» (Truchot, 2009). Ainsi, en France, une part de la planification de corpus est dévolue à la Délégation à la Langue française et aux Langues de France (DGLFLF) qui agit surtout au niveau terminologique. Lorsque le pouvoir agit sur l'emploi des langues, on parle de « planification de statut» (Truchot, 2009). Les exemples sont nombreux : le traité de VillersCotterêt (1539) sur l'emploi du français dans la justice, la loi Toubon (1994) règlementant l'emploi du français dans la sphère publique française, la Chartre de la langue française dite loi 101 au Québec garantissant l'emploi du français dans la province, la loi de 1995 sur la langue d'État de la République slovaque (Zákony pre l'udí, 1995) dont un des volets concerne l'emploi du slovaque dans la sphère publique.

La politique linguistique n'est cependant plus uniquement du ressort de l'État. Avec la mondialisation, des instances supranationales interviennent aussi sur les langues, surtout sur leur emploi (Truchot, 2009). Ainsi, le Conseil de l'Europe, en reconnaissant deux langues officielles (l'anglais et le français) et l'Union européenne trois langues de travail (allemand, anglais, français) favorisent de fait les langues de grande diffusion, même si la Charte européenne des langues régionales et minoritaires du Conseil de l'Europe tente de protéger les langues en position de fragilité en Europe.
L'internationalisation de la vie politique, économique et sociale pose donc de manière accrue la question de l'intervention du politique sur la diffusion des langues étrangères dans le cadre de la politique linguistique éducative.

\section{La politique linguistique éducative}

Face aux exigences accrues de compétitivité englobant progressivement tous les secteurs de la vie économique et sociale, la politique linguistique éducative, appliquée à l'enseignement des langues étrangères, vise surtout la performance. Grin (2005, p.9-10) fait la distinction entre l'efficience interne, liée au bon fonctionnement du système éducatif et à l'efficacité de l'enseignement, et l'efficience externe, liée aux facteurs extérieurs agissant sur l'enseignement des langues tels que les débouchés ou le rendement marchand. Il montre plus loin (2005, p.14) que la question de l'efficience externe s'inscrit d'abord dans le contexte de la construction européenne avec notamment l'appel de l'Union européenne au plurilinguisme par la maîtrise par les citoyens européens de deux langues étrangères de l'Union en plus de leur langue maternelle, politique initiée par le Livre blanc de l'Union européenne de 1995. Grin montre ensuite (2005, p.14) que cette question est directement liée au processus de mondialisation dont l'une des caractéristiques majeures est le besoin accru d'autonomie de l'individu à mesure que les institutions protectrices perdent de leur poids (État, corps intermédiaires, famille). Cette autonomie individuelle passe par la maîtrise des langues étrangères. Aussi, pour Truchot (2009), ce «recentrage sur l'individu » explique-t-il l'intérêt des politiques linguistiques actuelles pour le multilinguisme.

Les politiques linguistiques éducatives européennes sont donc amenées à répondre aux défis de la construction européenne et de la mondialisation.

\section{INSTITUTIONS EUROPÉENNES ET POLITIQUE ÉDUCATIVE}


Les deux acteurs majeurs de la construction européenne - le Conseil de l'Europe et l'Union européenne - ont mis en place des politiques linguistiques éducatives que nous nous proposons d'examiner à présent.

\section{Le Conseil de l'Europe}

\section{Les instruments d'intervention $d u$ Conseil de l'Europe sur les politiques linguistiques éducatives}

Parmi les textes majeurs et les multiples organisations mises en place par le Conseil de l'Europe depuis 1949, nous avons retenu cinq intruments agissant sur les politiques linguistiques éducatives des États membres.

Dès 1954, la Convention culturelle européenne encourage les pays signataires à promouvoir l'étude des langues afin de préserver le patrimoine européen et contribuer à une compréhension mutuelle entre les peuples. La Charte européenne des langues régionales et minoritaires de 1992 a eu pour conséquence la promotion de l'enseignement de certaines langues moins diffusées (Beacco, 2011). C'est aussi en fonction de la Charte, qu'elle signe en 2001, que la Slovaquie s'engage à œuvrer en faveur de l'enseignement des langues des minorités (Minedu, 2007, p.2). Le Centre européen des langues vivantes (CELV) ouvert à Graz en 1995 a influencé les pratiques linguistiques éducatives. Ainsi, Heyworth (2005, p.1) montre que «ses travaux ont une influence croissante sur les méthodes d'enseignement des langues en Europe » et ont contribué à la promotion de nombreux projets comme le Cadre européen commun de référence pour les langues (CECR), le Portofolio, l'éducation interculturelle («Miroirs et fenêtres») ou EMILE. Baranová (à paraître) constate que le CELV a favorisé l'ouverture sur l'extérieur des enseignants de langues étrangères slovaques qui «sont encouragés à entrer en contact avec le Centre européen pour les langues vivantes de Graz (Autriche) et à participer à des projets internationaux». Paru en 2001 et héritier des premiers dispositifs mis en place par le Conseil de l'Europe pour coordonner l'enseignement des langues étrangères - le projet « Langues vivantes » de 1963 et le Niveau seuil de 1975 (Truchot, 2014), le CECR est de loin l'instrument ayant le plus d'influence sur les politiques linguistiques éducatives. Il agit $\mathrm{d}$ 'abord au niveau du contenu et de l'organisation de l'enseignement des langues étrangères en proposant "une base commune pour l'élaboration de programmes de langues vivantes, de référentiels, d'examens, de manuels, etc. en Europe» (Conseil de l'Europe, 2001, p.9) en définissant des niveaux de compétence communs. Il influe aussi sur les pratiques $\mathrm{d}$ 'enseignement/apprentissage des langues étrangères par la promotion $\mathrm{du}$ plurilinguisme visant un enseignement/apprentissage décloisonné et plus autonome grâce au «Portofolio » ou «Portefeuille européen des langues» permettant à l'apprenant de gérer lui-même son apprentissage (Conseil de l'Europe, 2001, p.11). Enfin, l’Unité des politiques linguistiques du Conseil de l'Europe propose aux États membres l'élaboration de profils de leurs politiques linguistiques éducatives sous forme d'auto-évaluations accompagnées d'experts. Le rapport pour la Slovaquie élaboré entre 2004 et 2007 prévoit ainsi de fonder les épreuves de langues étrangères $d u$ nouveau baccalauréat entré en vigueur en 2004-2005 sur le CECR (Conseil de l'Europe, 2004-2007).

Le conseil de l'Europe dispose donc $d$ 'instruments efficaces pour mener une véritable politique linguistique éducative au service des orientations idéolologiques majeures de son projet européen. Les fondements idéologiques de la politique linguistique éducative du Conseil de l'Europe :

- Construire une Europe de la paix et de la démocratie :

Pour promouvoir en Europe la paix, la démocratie, les droits de l'homme et la diversité culturelle, la politique linguistique du Conseil de l'Europe suit trois axes. Le Conseil de l'Europe s'efforce d'abord de faciliter la mobilité européenne à travers la communication par la promotion de la méthode communicative dans l'enseignement des 
langues étrangères (Beacco, 2011). Le rapprochement des nations européennes passe aussi par la convergence des enseignements des langues étrangères grâce à la mise en place de référentiels communs comme le Niveau seuil (1975) puis le CECR (2001). Même si ces référentiels n'affirment $\mathrm{n}^{\prime}$ avoir aucun rôle contraignant, selon Beacco (2011), ils ont beaucoup contribué à rapprocher les systèmes d'évaluation et ont favorisé la généralisation des approches communicative puis actionnelle dans l'enseignement des langues étrangères. Baranová (à paraître) remarque ainsi qu'en Slovaquie «l'enseignement des langues étrangères lui-même est, dans son ensemble, conforme aux recommandations du Cadre commun de référence pour les langues ", en particulier en ce qui concerne les méthodes pédagogiques telles que l'approche communicative ou l'approche actionnelle par projets. Enfin, le troisième axe passe par la promotion du plurilinguisme face à l'apprentissage exclusif de l'anglais (Beacco, 2011). Baranová (à paraître) montre ainsi comment le cadre législatif slovaque de l'enseignement des langues étrangères s'est adapté aux perspectives du Conseil de l'Europe en matière de plurilinguisme avec la mise en place de «l'approche CLIL/EMILE (dont l'étape pilote en Slovaquie a été achevée en 2012, avec des résultats probants) et l'utilisation du Portofolio européen des langues ».

En œuvrant à la construction européenne par une coordination des politiques $\mathrm{d}$ 'enseignement des langues étrangères en Europe, la politique linguistique éducative du Conseil de l'Europe s'inscrit aussi dans un contexte idéologique plus global touchant depuis près de trente ans tous les secteurs de la vie collective, économique, sociale, politique et culturelle : la mondialisation.

- Répondre aux besoins de la mondialisation :

L'instrument qui tend à répondre le mieux aux besoins de la mondialisation en matière de politique linguistique éducative est sans conteste le CECR par son caractère global explorant tous les aspects de l'enseignement/apprentissage des langues étrangères et qui, pour cette raison, a tendance à jouer un rôle hégémonique sur les politiques linguistiques éducatives européennes.

\section{L'hégémonie du CECR sur les politiques linguistiques éducatives européennes}

Lefranc (2014) nous montre que l'hégémonie du CECR s'opère à tous les niveaux: dans les programmes d'enseignement des langues étrangères des pays européens - ce que nous démontrerons aussi plus loin pour la Slovaquie - et même ailleurs (en Algérie et au Japon, par exemple), dans les certifications comme celles du CIEP ou du CCIP en France ou de l'Examen élémentaire d'État de langue en Slovaquie, dans la recherche, car de nombreux travaux universitaires s'inspirent directement du CECR. Or, la validité du CECR ne semble pas être remise en cause, les critiques ne concernant que certaines lacunes et faiblesses. Aussi Lefranc (2014) critique-t-il l'absence de débat démocratique lors de l'adoption du CECR et explique ce quasi consensus en faveur du Cadre par le caractère non normatif affirmé du CECR qui est présenté comme un simple dispositif technique et, pour cette raison, « dépolitisé » et sans ambition idéologique apparente. Le parallèle entre l'évolution économique, sociale et politique de l'Europe et du monde sous l'effet de la mondialisation et le CECR paraît pourtant évident.

\section{CECR et mondialisation}

$$
\text { Selon Truchot }
$$

l'internationalisation de l'économie de l'Europe de l'Ouest dans les années 1960-70, marquée notamment par la création de la $\mathrm{CEE}$, a fait des langues étrangères une simple composante du marché devant répondre aux nouveaux besoins économiques. Les langues étrangères dans l'enseignement se sont alors vu attribuer une fonction nouvelle: traditionnellement au service de la formation littéraire et culturelle de l'individu, leur rôle est désormais essentiellement communicatif. Pour répondre à ce changement de 
paradigme, le Conseil de l'Europe se dote $\mathrm{d}$ 'instruments adaptés aux besoins nouveaux du marché linguistique: le projet «langues vivantes » (1963) puis le Niveau seuil (1975) qui aboutissent au CECR élaboré entre 1991 et 2001.

Ces orientations se sont précisées à la fin du XXe siècle. Avec l'avènement de la mondialisation dans les années 1990, l'enseignement, et en particulier l'enseignement des langues étrangères, est affecté par de nouvelles pratiques managériales, conséquences de la marchandisation $\mathrm{du}$ savoir. Pour Lefranc (2014), qui porte un regard très critique sur le Cadre, le CECR représente l'instrument par excellence de la rationalisation et de la standardisation de l'enseignementapprentissage des langues étrangères au service de l'économie de marché mondialisée. Sa première critique va à l'encontre du classement des compétences des apprenants en six niveaux - aspect du CECR qui connaît le plus grand succès - car cette nomenclature correspondrait parfaitement, selon lui, " aux normes de la nouvelle organisation scientifique $d u$ travail», du niveau C pour les cadres supérieurs au niveau A pour les besoins des emplois d'exécussion dans les services. Lefranc (2014) reproche aussi au CECR de proposer un modèle trop restrictif et trop utilitariste de formation en langues qui entérinerait la fusion entre les pratiques managériales d'entreprise et les pratiques d'enseignement. Ainsi, par le recours constant à l'évaluation/autoévaluation, mais aussi par les choix terminologiques renvoyant à la terminologie professionnelle (compétence, formateur, indicateur, mobilité, profil, etc.), le CECR aurait tendance à calquer l'enseignement-apprentissage des langues sur la gestion entrepreunariale.

Dans un premier temps, la politique linguistique éducative européenne semble donc avoir été du ressort du Conseil de l'Europe, la CEE se consacrant alors essentiellement à l'économie. Cependant, la Communauté européenne puis l'Union européenne s'affirmant comme organisation politique globale est elle aussi amenée à s'intéresser aux questions linguistiques.

\section{L'Union européenne}

\section{L'intérêt tardif de l'Union européenne} pour les politiques linguistiques éducatives

Le choix du multilinguisme amène la Commission européenne à mettre en place des instruments permettant de gérer le fonctionnement multilingue des institutions de la CEE : la base de données multilingue Euricautom (1975) puis le programme de recherche en traduction automatique Eurotra (1982). Pour garantir le multilinguisme, la CEE puis l'Union européenne doivent parallèlement encourager l'enseignement des langues étrangères en créant le programme Lingua (1989) chargé notamment de promouvoir les Modimes (les langues les moins enseignées et les moins diffusées) (Truchot, 2014). Pour promouvoir la mobilité au sein de l'espace unique européen, notamment la mobilité scolaire et universitaire et la formation professionnelle, sont mis en place les programmes Socrates (1995) et Léonard de Vinci qui n'ont pas d'objectifs spécifiquement linguistiques mais où les langues occupent de fait une place de choix (Truchot, 2014).

Ce n'est pourtant qu'à partir des années 1990 que l'Union européenne porte un intérêt véritable à la politique linguistique éducative en intégrant des volets linguistiques aux traités constitutifs. Voici la politique linguistique éducative de l'Union européenne :

- Les instruments de la politique linguistique éducative de l'Union européenne :

Depuis les années 1990, l'Union européenne tend à intervenir dans les politiques linguistiques nationales en faveur du multilinguisme désigné comme un facteur déterminant de la compétitivité européenne en se fixant comme objectif la maîtrise de deux autres langues de l'Union par chaque citoyen européen en plus de sa langue maternelle. Pour y parvenir, la Commission européenne met en place en 2005 un « indicateur européen des compétences linguistiques ", dispositif permettant aux États membres de "procéder aux ajustements nécessaires 
à leur politique d'enseignement et d'apprentissage des langues étrangères " (Eur-Lex, 2005). L'Union européenne possède ainsi une base juridique pour développer la dimension européenne dans les systèmes éducatifs des États membres, en encourageant notamment l'enseignement des langues étrangères: les articles 2 et 3 du Traité sur l'Union européenne (appelé également Traité de Maastricht, 1992) consolidés par le Traité de Lisbonne (2007), les articles 6 et 165 du Traité de fonctionnement de l'Union européenne (Parlement européen, 2015). Pour réaliser ses ambitions multilingues, l'Union européenne a mis en place des programmes dans le cadre de la stratégie Europe 2020 (2010) (Parlement européen, 2015): Erasmus+ qui comporte un programme de soutien linguistique en ligne et le volet Culture du programme Europe créative doté d'une composante linguistique.

Ainsi, en dépit de son principe de nonintervention dans les systèmes éducatifs des États membres selon lequel «dans l'Union européenne, l'éducation relève de la compétence des États membres et la Commission européenne ne peut intervenir dans ce domaine qu'au titre du principe de subsidiarité quand les dimensions et les effets de l'action envisagée impliquent que les objectifs poursuivis seront mieux réalisés au niveau communautaire », l'Union européenne s'est donné les moyens d'une véritable politique linguistique éducative. Cette intervention est particulièrement prégnante dans le cadre de la stratégie de Lisbonne qui a notamment marqué la réforme des programmes d'enseignement des langues étrangères en Slovaquie en 2007.

- La politique linguistique éducative de l'Union européenne et la stratégie de Lisbonne:

Pour répondre aux ambitions de la stratégie de Lisbonne définie en 2000 et visant à créer "l'économie de la connaissance la plus compétitive et la plus dynamique du monde " à l'horizon 2010, les ministres de l'Éducation des pays membres de l'Union européenne ont mis en place, en 2001, des objectifs communs à atteindre en matière de qualité, d'efficacité, d'accessibilité et d'ouverture des systèmes éducatifs (Charvet, 2007). Des dispositifs ont également été mis en place dans le cadre du programme «Éducation et formation 2010 » tels que le processus de Bologne (1999) pour assurer la convergence des systèmes d'enseignement supérieur, le processus de Copenhague (2002) pour la formation professionnelle et le programme pour l'Éducation et la formation tout au long de la vie (20072013) comprenant le programme Comenius pour l'enseignement élémentaire et secondaire. A cela s'ajoute le réseau Eurydice créé en 1980 pour favoriser les échanges d'informations entre les systèmes éducatifs européens et doté dans les années 1990 d'instruments comparatifs (rapports, évaluations). Lorsque Eurydice est intégré, en 1996, au programme Socrates, la Slovaquie rejoint le réseau avec d'autres futurs États membres. Depuis 2007, Eurydice est intégré au programme pour l'Éducation et la formation tout au long de la vie. Il constitue aujourd'hui une vaste base de données sur les sytèmes éducatifs européens avec des unités nationales dans les pays membres et une unité centrale à Bruxelles (Europe et formation, s.d.).

Les objectifs fixés par la stratégie de Lisbonne en matière de politique linguistique éducative apparaissent explicitement en Slovaquie dans les travaux préparatoires à la refonte des programmes de 2007. Ces travaux, qui répondent aux attentes du programme pour l'Éducation tout au long de la vie, préconisent ainsi un enseignement des langues étrangères favorisant la mobilité du citoyen sur le marché du travail et son autonomie par l'acquisition de compétences plutôt que de connaissances (Ministerstvo školstva Slovenskej republiky, 2007, p.3-4). Les objectifs de la politique linguistique de l'Union européenne sont aussi visibles dans le projet expérimental slovaque du CLIL 
(2008-2012) qui vise à faciliter la mobilité européenne sur le marché du travail, accroître la compétitivité ou promouvoir l'apprentissage tout au long de la vie (Menzlová, 2008, p.3).

Ainsi, le Conseil de l'Europe et l'Union européenne ont élaboré des politiques linguistiques éducatives orientées idéologiquement et inscrites dans l'évolution globale $\mathrm{du}$ monde d'aujourd'hui. Elles ont donné des moyens à leurs ambitions en se dotant d'outils efficaces leur permettant d'intervenir dans les politiques éducatives de leurs États membres. Pour mesurer l'incidence de ces interventions, nous prendrons l'exemple de la Slovaquie.

\section{ÉTUDE COMPARATIVE DE SES PROGRAMMES SCOLAIRES D'ENSEIGNEMENT DES LANGUES ÉTRANGÈRES DE 1997 ET DE 2007}

Pour évaluer l'évolution de la politique linguistique éducative de la Slovaquie au début du XXIe siècle, nous avons choisi de comparer les programmes scolaires d'enseignement des langues à l'école publique slovaque de 1997 et de 2007 dans la mesure où ils recouvrent une période cruxiale correspondant à l'intégration de la Slovaquie à l'Union européenne. En effet, alors qu'en 1997 la Slovaquie est membre du Conseil de l'Europe et affiche ses ambitions européennes, mais n'adhère pas encore à l'Union européenne, de 1997 à 2007, les évolutions survenues sont considérables, en Europe comme en Slovaquie : mise en place $\mathrm{du}$ processus de Lisbonne (2000), publication du CECR (2001) et adhésion de la Slovaquie à l'Union européenne. Pendant cette période, l'intégration européenne se renforce donc dans de nombreux domaines touchant l'enseignement des langues, si bien qu'en adhérant à l'Union européenne en 2004, la Slovaquie est amenée à adapter ses institutions nationales à ces évolutions. Dans cette perspective, les différences que nous pourrons observer entre les deux programmes nous permettrons d'évaluer l'influence des institutions européennes sur la politique linguistique éducative slovaque.
Les deux programmes scolaires d'enseignement des langues étrangères que nous analyserons ont été conçus par le Ministère de l'Éducation slovaque pour les écoles élémentaire (niveau ISCED 2) et secondaire (niveau ISCED 3). Les deux documents sont constitués de deux parties: une introduction présentant les objectifs pédagogiques principaux et un descriptif des contenus. Notre analyse suivra le même ordre : nous nous intéresserons d'abord aux objectifs puis nous décrypterons les contenus. Une différence significative, qui met en évidence l'incidence du CECR sur les programmes de 2007, apparaît d'emblée entre les deux dates dans le descriptif des contenus : alors que celui de 1997 suit le curriculum scolaire de la cinquième année de l'école élémentaire à la dernière année du lycée, celui de 2007 suit les niveaux de compétence du CECR, du niveau A1 au niveau $\mathrm{B} 2$.

Notre analyse des programmes sera thématique et comparative. Pour chaque programme, nous relèverons les thèmes principaux. Nous comparerons ensuite les thèmes de 1997 à ceux de 2007 en les regroupant en quatre catégories : les thèmes identiques en 1997 et en 2007, les thèmes ayant été modifiés en 2007, les thèmes figurant uniquement dans le programme de 1997 et ceux figurant uniquement dans le programme de 2007.

\section{Comparaison des objectifs des programmes}

Nous avons relevé deux objectifs identiques dans les deux programmes. D'abord, l'objectif essentiellement communicatif et interculturel de l'apprentissage des langues étrangères, la capacité à communiquer de l'apprenant devant s'appuyer sur la connaissance de la culture de l'autre et sa capacité à appréhender les cultures étrangères. Ensuite, l'interdisciplinarité des enseignements linguistiques: l'enseignement des langues étrangères est intégré au projet éducatif global de formation scolaire et contribue à la transmission de valeurs et au développement des aptitudes humaines de l'apprenant. 
Une différence importante entre les objectifs des deux programmes apparaît dans les références explicites au projet européen en 2007. Le programme de 2007 s'appuie ainsi systématiquement sur le CECR. En 2007, l'engagement européen est aussi évident dans les objectifs post-scolaires de l'enseignement des langues étrangères qui vise à préparer l'apprenant au marché du travail européen. En 1997, au contraire, l'enseignement des langues étrangères doit préparer l'apprenant à la vie professionnelle sans qu'il ne soit fait mention de sa dimension européenne.

L'ancrage européen du programme de 2007 semble aussi avoir contribué à réduire les ambitions éducatives de l'enseignement des langues étrangères concernant la formation de la personnalité de l'apprenant. La dimension humaniste et intellectuelle de l'enseignement des langues étrangères est ainsi encore affirmée dans le programme de 1997 où, au-delà des objectifs communicatifs et pragmatiques, la connaissance des langues étrangères doit aussi contribuer à l'enrichissement de la culture générale de l'apprenant. Y est souligné, par exemple, l'intérêt métalinguistique de l'enseignement des langues étrangères dans le but de susciter chez l'apprenant une réflexion sur le fonctionnement des langues. Cette dimension éducative est, au contraire, totalement absente du programme de 2007.

Cette différence semble s'expliquer par la volonté d'arracher l'enseignement des langues étrangères au cadre encore essentiellement scolaire du programme de 1997. En 2007, il ne s'agit plus uniquement de garantir la réussite scolaire puis professionnelle de l'apprenant, mais aussi de former un citoyen européen autonome et responsable, capable de s'orienter dans un monde de plus en plus complexe et dans lequel il doit faire preuve d'adaptabilité et de flexibilité.

L'affirmation de la dimension européenne des objectifs du programme de 2007 paraît donc évidente et s'exprime par une repésentation plus pragmatique de l'apprentissage des langues étrangères qu'en 1997. Cette évolution est également visible dans le choix des thèmes abordés.

\section{Comparaison des contenus thématiques} des programmes

D'une représentation humaniste à une représentation utilitariste de l'apprentissage des langues étrangères

Le glissement d'un enseignement des langues étrangères de tradition humaniste vers un enseignement utilitariste apparaît $\mathrm{d}$ 'abord dans la manière $\mathrm{d}$ 'aborder le thème de l'école. La différence dans l'intitulé du thème est significative : le thème "l'école " de 1997, confiné au milieu scolaire, devient «enseignement et travail» en 2007, ce qui marque une ouverture sur la vie professionnelle. Cette orientation plus pragmatique du programme de 2007 est renforcée par le choix du terme «enseignement», plus neutre et plus technique, de préférence à «école». On remarque aussi une centration sur la vie scolaire des sous-thèmes abordés en 1997 (« notre école», «l'emploi du temps », «mes camarades de classe », etc.) alors que ceux de 2007 (« l'école et son fonctionnement», « les matières scolaires», «le travail», «le système scolaire», "l'apprentissage tout au long de la vie», « les conditions de travail») font davantage le lien entre l'école et la vie professionnelle. Cette évolution semble liée à une nouvelle conception de l'enseignement/apprentissage qui met en valeur la notion d'apprentissage tout au long de la vie mise en avant dans les programmes de l'Union européenne. L'approche du thème de l'école dans le programme de 2007 reflète donc assez clairement la vision européenne d'une éducation ouverte sur le monde du travail et de la formation continue, ce qui ne semble pas encore être le cas dans le programme de 1997 qui ne comprend pas de thématique axée sur le monde du travail.

Le programme de 2007 comporte deux thèmes nouveaux qui marquent également cette orientation pragmatique de l'enseignement des langues étrangères. Ainsi, le thème «emploi » met l'accent sur l'utilité de l'enseignement des langues étrangères dans la perspective de l'intégration de l'apprenant sur le marché du travail européen. Le thème «la science et la technique au service de l'humanité » marque 
le lien entre l'enseignement des langues étrangères et la modernité technique.

Les thèmes figurant uniquement dans le programme de 1997 semblent, au contraire, davantage viser à la formation de la personnalité de l'apprenant. C'est le cas du thème « l'Europe et le Monde » explicitement lié à d'autres matières scolaires telles que l'éducation civique, la géographie et l'histoire, dans une optique interdisciplinaire afin de contribuer à la formation générale de l'apprenant. De même, le thème «le danger $\mathrm{du}$ racisme et de la haine entre nations, coopération et solidarité en vue d'y remédier » contribue à la formation éthique de l'apprenant. L'approche éthique de l'enseignement des langues étrangères n'est cependant pas absente dans le contenu du programme de 2007 mais elle est abordée de manière plus générale qu'en 1997 avec le thème "modèles et idéaux » et ses sousthèmes correspondants ( l'homme, ces modèles et idéaux», "modèles positifs et négatifs »).

\section{D'un enseignement des langues étrangères centré sur l'environnement immédiat de l'apprenant à un enseignement des langues étrangères ouvert sur le monde}

La plupart des thèmes équivalents dans les deux programmes se différencient par la manière dont ils sont abordés : en 1997, c'est l'environnement immédiat de l'apprenant et son cadre de proximité qui est privilégié; en 2007, l'optique est plus générale et le thème est abordé de façon à ouvrir l'apprenant sur le monde. C'est ainsi le cas du thème «la famille» de 1997, davantage tourné vers la vie proprement familiale de l'apprenant que le thème «famille et société » de 2007, également tourné vers la vie administrative et sociale. En effet, les sous-thèmes correspondants en 1997 (« les relations familiales», « les loisirs », « les fêtes familiales») mettent en valeur l'univers intime de l'apprenant alors que ceux de 2007 («nationalité/citoyenneté », «formulaires et documents», « religion») sont beaucoup plus ouverts sur le monde extérieur. En 2007, on peut donc observer une certaine déprivatisation, une neutralisation et une objectivation de l'approche du thème de la famille dans l'enseignement des langues étrangères en Slovaquie. L'apprenant est ainsi amené à prendre davantage de recul sur sa vie personnelle : on met en avant sa capacité à sortir de soi pour mieux s'adapter à un environnement différent du sien.

Nous avons noté la même différence $\mathrm{d}$ 'approche entre le thème "la maison », en 1997, et «la maison et le logis», en 2007. Dans l'ancien programme, les sous-thèmes correspondants sont tournés vers la vie intime de l'apprenant (« notre appartement / notre maison », « notre ville / notre village», «le milieu dans lequel je vis», «ma chambre»), alors que dans le nouveau programme, ils sont plus généraux ("société et environnement», "société et style de vie») et concernent aussi bien la Slovaquie que d'autres pays. Ici aussi, en 2007, l'apprenant est donc encouragé à s'adapter aux autres cultures en sortant du strict cadre slovaque.

Nous avons retrouvé les mêmes différences d'approche, plus personnelle en 1997 et plus neutre en 2007, dans les autres thèmes proposés. Par exemple, le thème du voyage est abordé en 1997 à partir de l'expérience personnelle de l'apprenant, comme nous l'indiquent les sous-thèmes ("où j'aime aller», "les pays que j'ai visité »), alors que les sous-thèmes de 2007 sont centrés sur les moyens de transport et le tourisme. De même, le thème «notre partrie » en 1997 met explicitement en valeur le pays de l'apprenant avec un certain accent patriotique alors que le thème correspondant de 2007, « la Slovaquie », présente le pays de manière beaucoup plus neutre.

\section{CONCLUSION}

Ainsi, le Conseil de l'Europe et l'Union européenne ont progressivement mis en place une véritable politique linguistique éducative, en phase avec les orientations politiques, économiques et sociales de la mondialisation, que les États membres sont incités à adopter. L'évolution des programmes scolaires slovaques vers une efficience accrue de l'enseignement des langues étrangères visant explicitement à 
développer l'aptitude de l'apprenant à s'adapter au marché européen du travail confirme cette tendance. Ce phénomène semble même largement dépasser le domaine de l'enseignement des langues. En effet, certaines innovations récentes du système éducatif slovaque vont dans le même sens comme, par exemple, l'introduction il y a près de dix ans du test de niveau Komparo auquel sont soumis les élèves de quatrième année de l'école élémentaire et réalisé par une institution privée indépendante du Ministère de l'Éducation nationale slovaque dans le but de comparer le niveau des écoles et d'évaluer les connaissances et aptitudes des élèves. Le rapprochement avec les pratiques managériales du secteur privé est ici tentant, car sur certains points évaluation des établissements d'enseignement, appel à un prestateur de service externe - ce test de niveau s'apparente à un véritable audit du premier cycle de l'enseignement élémentaire slovaque (niveau ISCED 1 ).

\section{REMERCIEMENTS}

Je remercie Madame Elena Baranová de m'avoir fait profiter de sa riche expérience en didactique du FLE et pour ses précieux conseils concernant la situation en Slovaquie.

\section{RÉFÉRENCES}

Baranová, E. (sous presse). Plurilinguisme dans les écoles slovaques. Colloque international. Politique et idéologie en didactique des langues: acteurs et discours. Paris, France: INALCOPLIDAM.

Beacco, J.-C. (2011). Les orientations du Conseil de l'Europe en matière de politique linguistique. Actes $d u$ séminaire "L'enseignement des langues vivantes ». Direction de l'enseignement scolaire-CRDP de Versailles : http://eduscol.education.fr/cid46542/ac tes-seminaire-enseignement-deslangues-vivantes-perspectives27-mars2001.html

Charvet, P. (2007). La politique de l'Union européenne en matière d'éducation et de formation. Sèvre, France: CIEP. http://www.ciep.fr/ressources/politiqu e-lunion-europeenne-matiere-

deducation-formation-decembre-2007

Conseil de 1'Europe (2001). Cadre européen commun de référence pour les langues.

Strasbourg, France: Unité des politiques linguistiques. http://www.coe.int/t/dg4/linguistic/So urce/Framework_FR.pdf

Conseil de 1'Europe (2004-2007). Profil de la politique linguistique éducative. République slovaque. Strasbourg, France: Conseil de l'Europe. https://www.coe.int/t/dg4/linguistic/pr ofils1_FR.asp

Eur-Lex (2005). L'indicateur européen des compétences linguistiques. http://eurlex.europa.eu/legalcontent/FR/TXT/?uri=URISERV\%3Ac1 1083

Europe et Formation. (s.d.). Réseau Eurydice. Repéré à http://www.europe-etformation.eu/eurydice,5129.html

Grin, F. (2005). L'enseignement des langues étrangères comme politique publique. Avis du Haut Conseil de l'évaluation de l'école. Paris, France: DEP/Bureau de l'édition. Ministère de l'Éducation nationale, de l'Enseignement supérieur et de la Recherche. http://www.ladocumentationfrancaise. $\mathrm{fr} /$ var/storage/rapportspublics/054000678.pdf

Heyworth, F. (2005). Les dix premières années du Centre européen pour les langues vivantes à Graz - sa contribution à l'enseignement des langues. Graz, Autriche: Centre européen pour les langues vivantes. Repéré à http://www.ecml.at/Portals/1/docume nts/about-us/article_Heyworth_FR.pdf Lefranc, Y. (2014). Le management enchanteur: gouvernement, technologie et double langage du CECRL. Les Cahiers du GEPE, 6. Repéré à

http://www.cahiersdugepe.fr/index.ph p?id=2694

Minedu. (2007). Návrh koncepcie výchovy a vzdelávanie národnostných menšín. Repéré https://www.minedu.sk/data/att/948.p df 
Ministerstvo školstva Slovenskej republiky (2007). Koncepcia vyučovania cudzich jazykov v základných a stredných školách. Repéré

http://www.rokovania.sk/File.aspx/Vie wDocumentHtml/Mater-Dokum51587? prefixFile $=\mathrm{m}_{-}$

Menzlová, B. (2008). Didaktická efektívnost' metódy CLIL na proom stupni ZŠ vo vyučovaní cudzich jazykov. Repéré à http://www.statpedu.sk/sites/default/fi les/dokumenty/vyskumne-ulohyexperimentalne-

overovania/projekt_didakticka_efektiv nost_metody_clil.pdf

Parlement européen (2015). La politique linguistique. Repéré à http://www.europarl.europa.eu/aty ourservice/fr/displayFtu.html?ftuId=F TU_5.13.6.html

Puren, Ch. (1989). L'enseignement scolaire des langues vivantes étrangères en France au XIXe siècle ou la naissance d'une didactique. Langue française. 82,
8-19. Repéré à

http://www.persee.fr/doc/lfr_0023-

8368_1989_num_82_1_6377]

Truchot, C. (2009). L'analyse des pratiques d'évaluation des politiques linguistiques: un objet d'étude à constituer. Les Cahiers du GEPE, 1. Repéré à http://www.cahiersdugepe.fr/index.ph $\mathrm{p}$ ?id=686\#citation

Truchot, C. (2014). Le contexte politique et économique du CECR et les politiques linguistiques européennes. Les Cahiers $d u \quad$ GEPE, $6 . \quad$ Repéré à http://www.cahiersdugepe.fr/index.p hp?id=2685\#citation

Zákony pre l’udí. (1995). Zákon Národnej rady Slovenskej republiky o štátnom jazyku Slovenskej republiky. Repéré à http://www.zakonypreludi.sk/zz/1995270

Zarate, G. (2010). Représentations de l'étranger et didactique des langues. Paris, France: Didier. 